\title{
Caracterización del dolor perineal postparto en puérperas
}

\author{
Characteristics of perineal pain postpartum in women
}

Paula Andrea Medina-Piedrahita1, Crystian Borrero-Cortés², Pedro José Herrera-Gómez³, Juan Manuel Ospina-Diaz ${ }^{4}$

1 MD. Esp. en Anestesiología y Reanimación. Universidad Nacional de Colombia. Bogotá, Colombia. e-mail: paulamedp@yahoo.com

2 MD. Residente II Anestesiología y Reanimación. Universidad Nacional de Colombia. Bogotá, Colombia. e-mail: crystianborreroc@gmail.com

3 MD. Esp. en Anestesiología y Reanimación. Profesor Asistente Facultad de Medicina, Universidad Nacional de Colombia. Bogotá, Colombia. e-mail: pjherrerag@unal.edu.co

4 MD. MSc. Epidemiología. Profesor Titular Universidad Pedagógica y Tecnológica de Colombia. Tunja, Colombia. e-mail: juan.ospina@uptc.edu.co

Fecha de recepción: Mayo 25 - 2016 Fecha de revisión: Septiembre $1^{\circ}$ - $2016 \quad$ Fecha de aceptación: Diciembre 12 - 2016

Medina-Piedrahita PA, Borrero-Cortés C, Herrera-Gómez PJ, Ospina-Diaz JM. Caracterización del dolor perineal postparto en puérperas de Bogotá, Colombia. 2013. Univ. Salud. 2016;18(3):556-565. DOI: http://dx.doi.org/10.22267/rus.161803.61

\section{Resumen}

Introducción: El dolor perineal posparto es un problema clínico frecuente, no abordado en profundidad en Colombia. Objetivo: Determinar la prevalencia, severidad y posibles factores asociados con el dolor perineal postparto en una muestra de mujeres en el Hospital La Victoria-Instituto Materno Infantil de Bogotá. Materiales y métodos: Estudio observacional prospectivo. Se evaluó el grado de dolor perineal percibido en 133 pacientes en posparto, mediante la escala análoga numérica de dolor, estando acostada, sentada, caminando y yendo al baño (micción y/o defecación), a las 12 y 48 horas postparto. Datos demográficos, del parto y recién nacido fueron tomados directamente de las historias clínicas. Resultados: Prevalencia global del dolor perineal moderado a severo 36-39,5\% dependiendo de la actividad a las 12 horas del parto y entre $28-31,6 \%$ a las 48 horas. El dolor perineal se encontró asociado, en cualquiera de las actividades valoradas, con el uso de analgesia epidural durante el trabajo de parto, desgarro grado II o mayor, peso y perímetro cefálico del recién nacido. Se registra mejor efecto analgésico con dosis de acetaminofén de $3 \mathrm{~g}$ diarios o más. Conclusiones: El dolor perineal moderado a severo afecta a menos del $50 \%$ de las mujeres postparto vaginal; se asocia con la frecuencia y magnitud del trauma perineal. Los resultados sugieren la necesidad de profundizar en el estudio del manejo analgésico más adecuado para estas pacientes.

Palabras clave: Período de postparto; dolor; analgesia obstétrica. (Fuente: DeCS, Bireme).

\begin{abstract}
Introduction: Postpartum perineum pain is a common clinical problem, which is not addressed in detail in Colombia. Objective: To assess prevalence, magnitude and possible factors associated with postpartum perineum pain in a sample of women in the Hospital "La Victoria-Instituto Materno Infantil", in Bogotá. Materials and methods: A prospective observational study was performed where the degree of perineum pain perceived by 133 postpartum patients was evaluated by using numerical analog scale of pain when they were sitting down, lying, walking and going to the bathroom (urination and/or defecation), at 12 and 48 hours of postpartum . Demographic, birth and newborn data were taken directly from the medical records. Results: Overall prevalence of moderate to severe perineum pain
\end{abstract}


36 to $39.5 \%$ depending on the activity at 12 hours of delivery, and since 28 to $31.6 \%$ at 48 hours after it. Perineum pain was associated, during any of the activities assessed, with the use of epidural analgesia during labor, laceration of degree II or greater, birth weight and head circumference of the newborn. Better analgesic effect of acetaminophen doses as $3 \mathrm{~g}$ per day or more was registered. Conclusions: Moderate to severe perineum pain affects less than $50 \%$ of women with vaginal postpartum. It is associated with the frequency and degree of perineum trauma. The results suggest the need for further study of the most appropriate analgesic doses for these patients.

Keywords: Postpartum period; pain; obstetrical analgesia. (Source: DeCS, Bireme).

\section{Introducción}

El evento de ocurrencia del parto es un intrincado proceso que involucra innumerables modificaciones orgánicas fisiológicas y bioquímicas, que se traducen en una respuesta adaptativa de tipo neuroendocrino; la percepción el dolor constituye una parte de este complejo proceso. El estado de estrés ocasionado por el dolor estimula la síntesis y liberación de hormona adrenocorticotropa (ACTH), y a través de ella de $\beta$-Endorfina, como un sistema modulador de analgesia endógeno ${ }^{1}$. No obstante, durante el postparto, el dolor puede mantenerse e incluso incrementarse como consecuencia de los eventos propios del parto como los desgarros y traumas de tejidos blandos, y por factores como paridad, práctica de episiotomía, instrumentación del parto ${ }^{1 .}$

Aunque el dolor perineal y la dorsalgia baja se han considerado como situaciones inevitables que acompañan al postparto, para algunas mujeres del dolor puede ser debilitante, e interferir con el sueño y las actividades cotidianas propias de la maternidad; dolor dorsal bajo ha sido reportado entre 50 a $80 \%$ de las mujeres gestantes ${ }^{2}$. Un estudio en Canadá encontró que de 447 mujeres que dieron a luz por vía vaginal, la prevalencia total de dolor perineal fue de $92 \%$ al primer día, $67 \%$ al día séptimo y de $7 \%$ a las seis semanas 3 .

En Australia, East et al., hallaron una prevalencia similar de dolor perineal postparto (90\%), percibiendo el $37 \%$ de ellas dolores de intensidad moderada y severa que interfirieron con la movilización, la lactancia, el cuidado del neonato y el sueño ${ }^{4}$. Excepcionalmente, el estudio realizado en Brasil por Amorim y colaboradores encontraron una prevalencia baja en comparación con la reportada en otros trabajos 5 ; se destaca en este estudio el hecho de que el dolor generado por el trauma del parto, puede interferir significativamente en el proceso normal de amamantamiento al ocasionar fenómenos hormonales que alteran la producción de la leche materna ${ }^{6}$.

Los estudios adelantados sobre los factores probablemente más relacionados con la aparición de dolor agudo después del parto han perfilado a la intensidad y gradación del trauma perineal como el evento mayormente asociado con el dolor perineal postparto. La magnitud del trauma varía desde el micro-trauma, desgarros perineales y laceraciones vaginales leves, hasta las episiotomías para-medianas y los desgarros perineales y vaginales de mayor grado ${ }^{3,4}$. La prevalencia de trauma perineal, aunque variable, es común entre las madres en el postparto inmediato. Se han reportado prevalencias de 39$49 \%$ de trauma perineal que requirió sutura en mujeres australianas y hasta del $70 \%$ en Brasil, dado que, en esta región, así como en la mayoría de países de Latinoamérica, se siguen practicando episiotomías de rutina en los partos normales ${ }^{7}$.

La presencia de dolor de intensidad con significado clínico, varía desde el primero al séptimo día después del parto, en diferente proporción y en relación directa con la magnitud del trauma ocurrido durante el parto; de tal manera, que la prevalencia de dolor postparto en mujeres que no sufrieron trauma considerable fue de $75 \%$ en el primer día y de $38 \%$ en el séptimo, mientras que en las mujeres con desgarros perineales de primero y segundo grado, la percepción de dolor en el mismo período ocurrió en $95 \%$ y $60 \%$ respectivamente 3 . 
El manejo del dolor desencadenado durante el puerperio es en la actualidad objeto de controversia entre los especialistas, de manera que coexiste un apreciable número de alternativas, algunas de amplia utilización en Colombia y otras más restringidas. En la literatura relacionada con la terapia farmacológica, la más utilizada es el acetaminofén, seguido en frecuencia de algunos antiinflamatorios no esteroideos (AINES) como el ibuprofeno8; los opiáceos sólo se emplean en ocasiones muy particulares, de manera que su uso es restringido. Algunas de las terapias alternativas ofrecidas para el manejo de este tipo de dolor incluyen aplicación de frío y/o calor local, acupuntura, terapia neural, aromaterapia, y diversas técnicas de hipnosis y relajación 9 .

Una primera aproximación al problema del dolor postparto, consiste en la elaboración de estudios observacionales para establecer los factores asociados con el parto y con el neonato y su relación con las labores que impone el cuidado del recién nacido, su incidencia y magnitud. Es claro que a la luz de la epidemiología, es importante contar con estadísticas propias que permitan conocer el comportamiento de un evento dado. Con ésta información podrán diseñarse estudios clínicos controlados sobre técnicas y manejos analgésicos en el postparto; en consecuencia, el presente estudio se formuló con el propósito de caracterizar el dolor perineal postparto, mediante la estimación de la prevalencia, magnitud y factores probablemente relacionados, tanto en condiciones de reposo (acostada, sentada) como de movilización (caminar, ir al baño), en una muestra de mujeres que tuvieron partos vaginales en el Instituto Materno Infantil de Bogotá, Colombia (2013).

\section{Materiales y métodos}

Se diseñó un estudio observacional, longitudinal prospectivo, de cohorte única. Se definió como población general a las mujeres en condición de postparto de la ciudad de Bogotá, Colombia, como población objetivo se consideró a las mujeres que asisten al parto en el Hospital de la VictoriaInstituto Materno Infantil. Se calculó en el programa Epidat3 una muestra de 122 mujeres, considerando un nivel de significancia alfa de 0,05 una prevalencia de dolor perineal postparto, de magnitud moderado a severo (mayor de 4 en la escala análoga del dolor), del $50 \%$ y una relación de 1,66 entre las mujeres que reciben analgesia epidural respecto de las que no la reciben durante el trabajo de parto. Las pacientes fueron vinculadas al estudio mediante muestreo secuencial aleatorio por conveniencia.

Para el estudio se incluyeron casos incidentes captados en los servicios clínicos del Hospital La Victoria-Instituto Materno Infantil, institución de IV nivel de complejidad que absorbe demanda proveniente del centro oriente de la ciudad de Bogotá, lo que en cierta medida garantiza la representatividad de la muestra seleccionada. Como criterios de inclusión se consideró tipo de parto vaginal, edad entre 14 y 45 años y posibilidad de seguimiento durante al menos 48 horas después del parto, se excluyeron pacientes con manifiesta incapacidad cognitiva o no dispuestas a proporcionar la información requerida. Acorde con la Resolución 8430 de 1993 Artículo 11, literal A y Artículo 16, Parágrafo primero, se clasificó a esta investigación como un estudio sin riesgo, razón por la cual no fue necesaria la obtención de consentimiento informado escrito por las mujeres participantes.

La variable de salida, magnitud del dolor se estimó utilizando la escala análoga del dolor; la primera versión de este instrumento fue introducida por Downie en $1978^{10}$, y ha sido satisfactoriamente evaluada en tiempo reciente, reflejando una alta capacidad discriminatoria para diferenciar el dolor subyacente de los picos de dolor y también una alta reproducibilidad (Kappa de Cohen 0,86), comparada con las escalas visuales del dolor ${ }^{11}$. Mediante este instrumento las pacientes clasifican la magnitud del dolor en valores numéricos que van de 0 a 10 , siendo 0 la ausencia de dolor y 10 el máximo dolor imaginable.

Se definió como dolor en reposo aquel que se presenta mientras la materna, durante el posparto, manifieste percibir en la escala análoga, dolor perineal estando acostada o sentada y dolor 
en movimiento aquel que se presenta al caminar o cuando realiza micción o defecación; para efectos del estudio se definió el valor 0 como ausencia de dolor, 1 a 3 dolor leve, 4 a 6 dolor moderado y valor igual o superior a 7 dolor severo.

Como covariables se registraron: la edad, número de semanas de gestación, tipo de parto y procedimientos realizados, ocurrencia y grado de desgarro, práctica de episiotomía, resultado final, peso, talla, perímetros cefálico y torácico del recién nacido desde la Historia clínica de cada una de las pacientes incluidas en el estudio.

Transcurridas 12 y 48 horas después del parto se exploró en cada paciente la magnitud del dolor percibido, en cada una de las cuatro condiciones o momentos definidos en el protocolo, los correspondientes valores fueron consignados en el instrumento. Respecto de la calidad de la información se anota que ella fue obtenida por una profesional de la medicina previamente entrenada y con conocimientos avanzados sobre el tema del dolor.

Con la información obtenida, se construyó una base de datos, que se validó para detectar y corregir posibles errores de digitación en el programa VALIDATE Epi-Info v 6-04d.

El procesamiento de la información incluyó un análisis univariado que permitió examinar las características del grupo de pacientes, y determinar su comparabilidad en una línea de base. Cada variable se exploró en su correspondiente nivel de medición y luego se describió en forma de medias para las variables continuas y proporciones para las categóricas o nominales; todas se reportaron con sus intervalos de confianza (IC), con un nivel alfa de 0.05. La comparación entre grupos se realizó a través de pruebas $\mathrm{t}$ de Student, ANOVA y Chi cuadrado cuando se consideró pertinente.

Posteriormente, se adelantó un análisis exploratorio bivariado, el cual permitió establecer la asociación de las diferentes covariables, con la variable de salida dolor perineal, durante 4 condiciones diferentes (acostada, sentada, caminando, en el baño). Esto fue realizado mediante el cálculo de medidas de efecto absoluto; Odds Ratio (OR) dada la exposición y sus respectivos IC al 95\%, junto con el cálculo de los valores p obtenidos en la comparación de medias o de los correspondientes promedios, siendo la hipótesis nula la de no diferencia entre los grupos. Se realizó un análisis estratificado de manera exploratoria, con el fin de identificar posibles variables confusoras. El procesamiento de variables y el análisis estadístico fue realizado utilizando el programa Epi-Info7 (CDC/EpiInfo, versión 7. Epidemiología en ordenadores. Atlanta, Georgia).

\section{Consideraciones éticas}

El protocolo de investigación fue aprobado por el Comité de Bioética en Investigaciones del Hospital La Victoria-Instituto Materno Infantil, acotando que acorde con lo consignado en la Resolución 8430 de 1993, ya mencionada, se clasificó como un estudio sin riesgo, razón por la cual no fue necesaria la obtención de consentimiento informado escrito.

\section{Resultados}

\section{Caracterización de la muestra}

La muestra definitiva estuvo conformada por 133 mujeres, con edad promedio de 21,3 años $(\mathrm{SD}=4,9) ; 51,2 \%$ de las pacientes registró 20 años o menos de edad, mientras que el resto está entre los 21 y los 41 años; 70,68\% (n=94) eran primíparas, $19,55 \%$ cursaban su segundo parto y el resto eran multíparas $(9,78 \%) ; 4,51 \%(n=6)$ eran afrocolombianas y el resto mestizas.

Respecto de los partos, la mayoría fueron normales; $3,01 \% \quad(n=4) \quad$ debieron ser instrumentados; sólo en un caso se consideró expulsivo prolongado; en 4 partos $(3,01 \%)$ ocurrió desgarro para-uretral, mientras que en 77 partos $(57,89 \%)$ hubo desgarro; de estos 77 casos, la mayoría de desgarros se clasificó como grado II $(64,94 \%), 33,77 \%$ desgarro grado I y apenas 1,3\% desgarros grado III; la mayoría de los desgarros $(n=66)$ fue suturado. Con excepción de una paciente sometida a analgesia peri-dural, el resto fue medicado en el postparto con 
acetaminofén, 53 pacientes $(39,85 \%)$ recibieron 2 o menos gramos por día, mientras que el resto (59,39\%) fueron medicadas con 3 o $4 \mathrm{~g} /$ día.

A las 12 horas del parto, dolor moderado a severo en $36,1 \%$ de las mujeres en posición acostada y en $39,5 \%$ sentada. A las 48 horas el dolor estuvo presente en $31,6 \%$ en posición acostada y $28,7 \%$ sentadas. Respecto del dolor incidente, asociado a la práctica de alguna actividad como caminar o ir al baño, ocurrió dolor en 38,3\% a las 12 horas y en $28,7 \%$ a las 48 horas.

Respecto del valor promedio de la magnitud del dolor experimentado, este se registra en la figura 1.

Figura 1. Nivel promedio de dolor perineal postparto en puérperas, Bogotá 2013

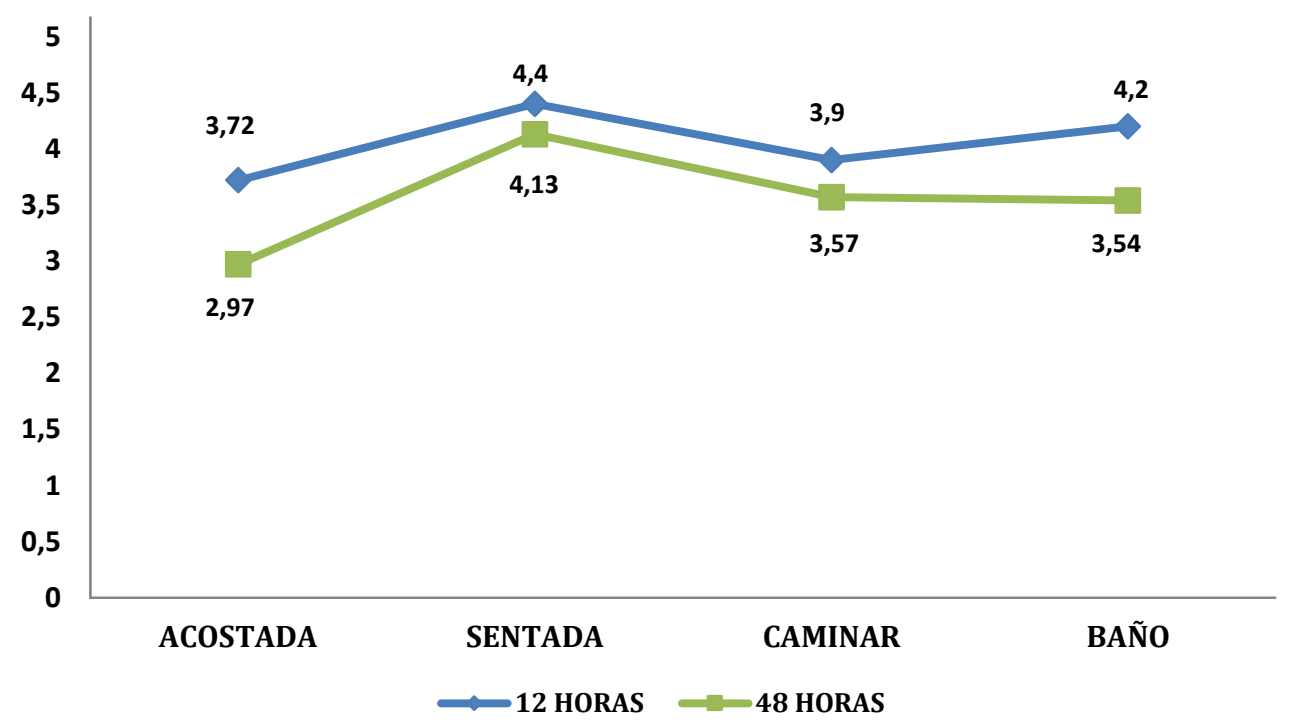

No se aprecian diferencias estadísticamente significativas en el grado de percepción del dolor a las 12 y a las 48 horas; este se percibe un poco más intenso cuando las mujeres están sentadas y cuando deben ir al baño.

En la figura 2 se muestra el nivel promedio de percepción del dolor, discriminando el grupo según si hubo o no desgarro perineal; se puede evidenciar que el desgarro es un evento que acentúa la percepción del dolor; también que la posición sentada en reposo y el acto de ir al baño se reiteran como momentos en los que ocurre dolor con mayor intensidad, tanto transcurridas 12 horas como a las 48. Además, es evidente que el grado de dolor disminuye con el tiempo, aunque las diferencias no son significativas en su totalidad.

El empleo de analgesia epidural durante el trabajo de parto no se tradujo en una reducción significativa de percepción del dolor, aunque se registraron diferencias significativas en el reposo y al ir al baño 48 horas después del parto (Figura $3)$. 
Figura 2. Media de percepción del dolor perineal postparto según desgarro. IMI 2013

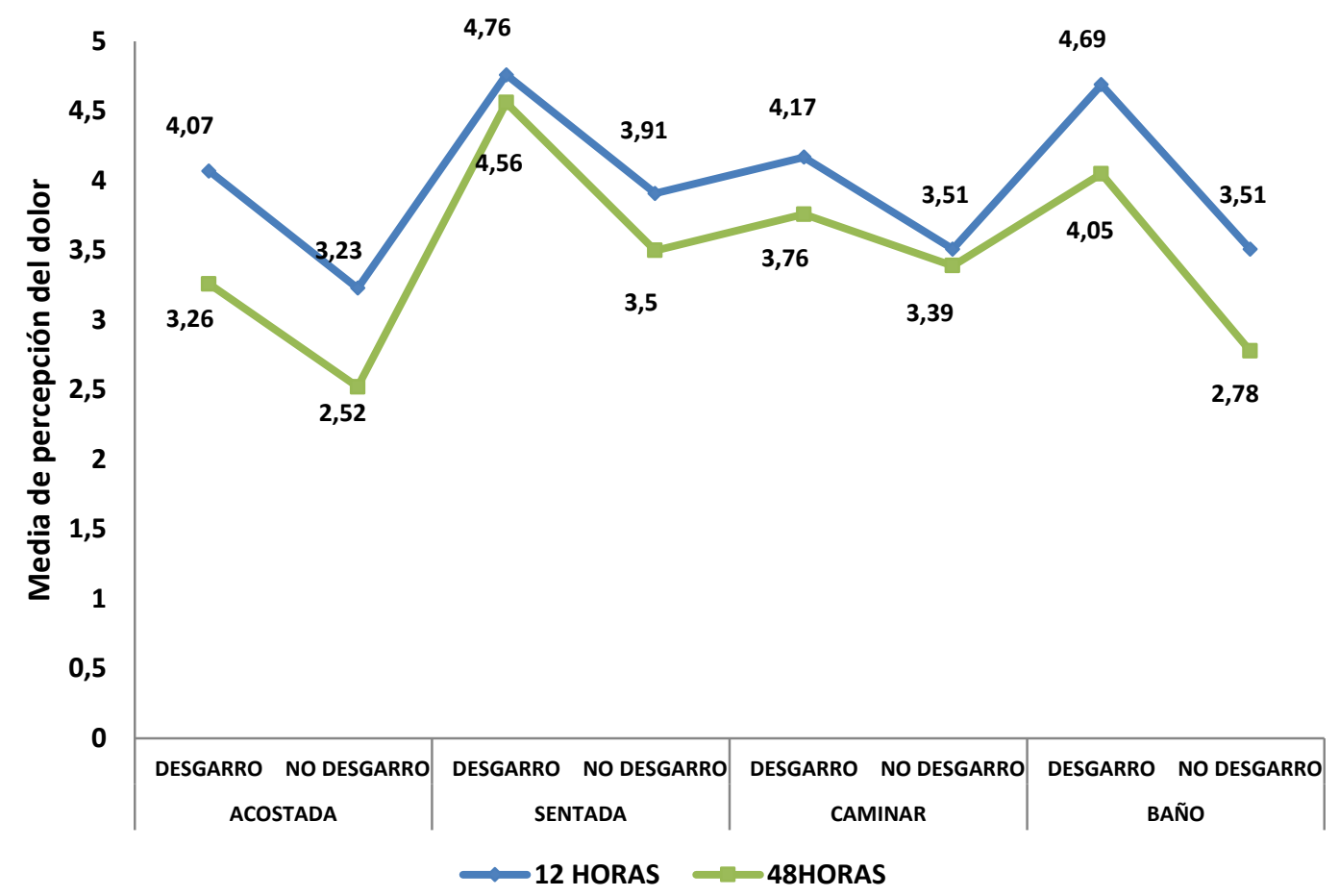

Figura 3. Media de percepción dolor perineal Según uso de analgesia epidural en T de parto a 12 y 48 horas. IMI 2013

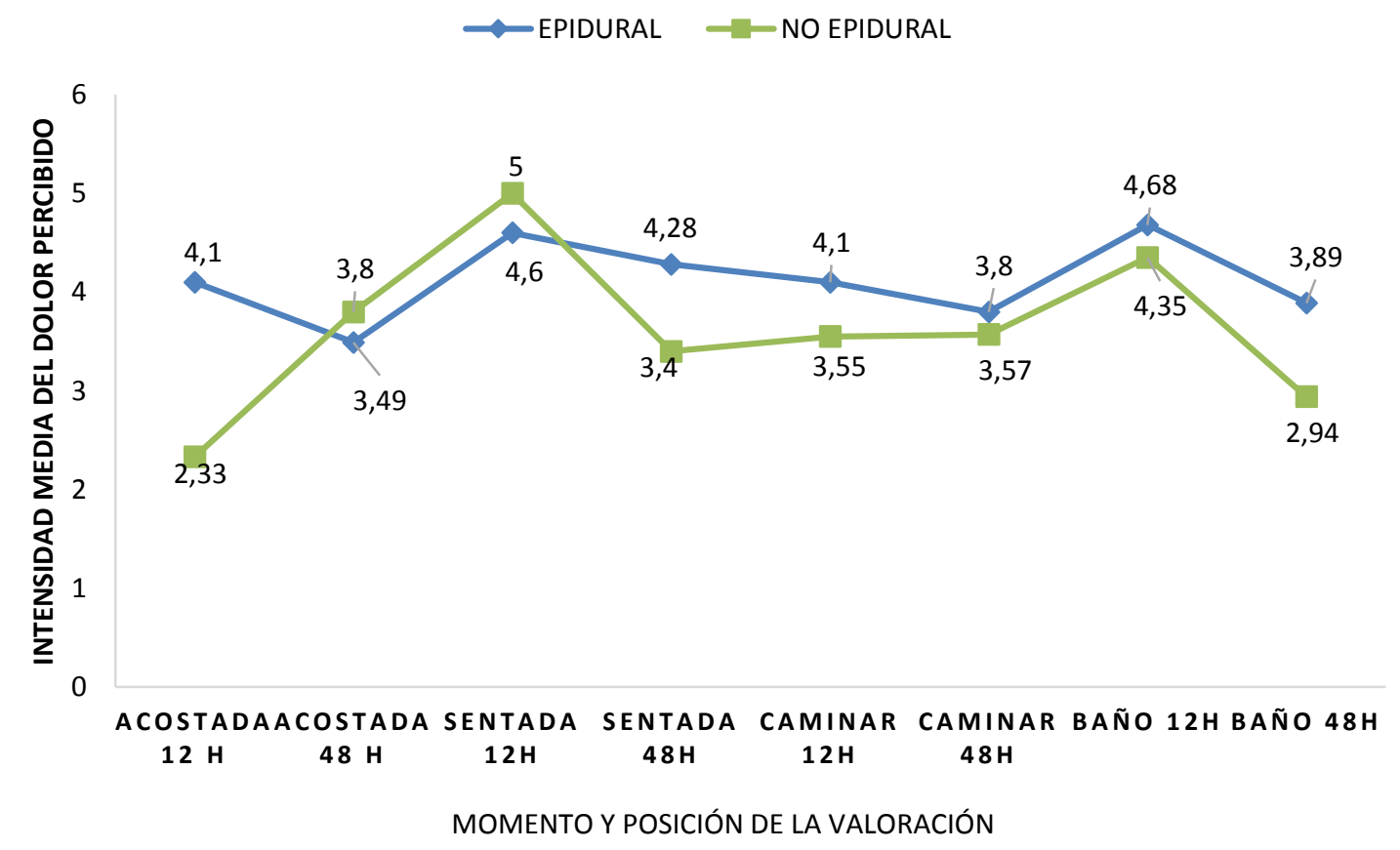


En la tabla 1 se muestra la significancia de la fuerza de asociación entre la variable de salida dolor perineal y algunos factores del trabajo de parto. Se encuentra que el uso de analgesia epidural durante el trabajo de parto puede incrementar la probabilidad de percepción del dolor perineal tanto a las 12 como a las 48 horas del post parto. También se encontró significancia entre el dolor perineal postparto, la ocurrencia de desgarros perineales grado II o mayor, la sutura de estos, el peso fetal mayor de $3500 \mathrm{~g}$ y el perímetro cefálico mayor a 35 centímetros.

Tabla 1. Factores asociados con la percepción del dolor perineal Post-Parto. IMI Bogotá, 2013

\begin{tabular}{|c|c|c|c|c|c|c|c|}
\hline \multirow{2}{*}{ Condición } & \multirow{2}{*}{ Variable } & & \multicolumn{2}{|c|}{ Dolor $>4$} & \multirow{2}{*}{$\mathbf{O R}$} & \multirow{2}{*}{ IC $95 \%$} & \multirow{2}{*}{$\mathbf{p}^{*}$} \\
\hline & & & $\mathbf{S i}$ & No & & & \\
\hline \multirow{6}{*}{$\begin{array}{l}12 \text { Horas } \\
\text { acostada }\end{array}$} & \multirow[t]{2}{*}{ Analgesia epidural } & $\mathrm{Si}$ & 26 & 24 & \multirow{2}{*}{2,01} & \multirow{2}{*}{$0,99-4,12$} & \multirow{2}{*}{0,028} \\
\hline & & No & 29 & 54 & & & \\
\hline & \multirow[t]{2}{*}{ Desgarro suturado } & $\mathrm{Si}$ & 33 & 33 & \multirow{2}{*}{4,0} & \multirow{2}{*}{$0,78-20,3$} & \multirow{2}{*}{0,04} \\
\hline & & No & 2 & 8 & & & \\
\hline & \multirow[t]{2}{*}{ Desgarro grado II o mayor } & $\mathrm{Si}$ & 29 & 22 & \multirow{2}{*}{3,57} & \multirow{2}{*}{$1,27-10,0$} & \multirow{2}{*}{0,007} \\
\hline & & No & 7 & 19 & & & \\
\hline \multirow[t]{4}{*}{12 Horas baño } & \multirow[t]{2}{*}{ Desgarro } & $\mathrm{Si}$ & 41 & 34 & \multirow{2}{*}{2,22} & \multirow{2}{*}{$1,08-4,56$} & \multirow{2}{*}{0,015} \\
\hline & & No & 19 & 35 & & & \\
\hline & \multirow[t]{2}{*}{$\mathrm{RN}$ con peso $>3500 \mathrm{~g}$} & $\mathrm{Si}$ & 10 & 4 & \multirow{2}{*}{3,21} & \multirow{2}{*}{$0,95-10,86$} & \multirow{2}{*}{0,029} \\
\hline & & No & 49 & 63 & & & \\
\hline \multirow{2}{*}{$\begin{array}{l}48 \text { Horas } \\
\text { acostada }\end{array}$} & \multirow[t]{2}{*}{ Analgesia epidural } & $\mathrm{Si}$ & 17 & 24 & \multirow{2}{*}{2,76} & \multirow{2}{*}{$1,11-6,86$} & \multirow{2}{*}{0,014} \\
\hline & & No & 11 & 43 & & & \\
\hline \multirow[t]{2}{*}{48 Horas sentada } & \multirow[t]{2}{*}{ Analgesia epidural } & $\mathrm{Si}$ & 23 & 18 & & & \\
\hline & & No & 18 & 36 & & ט, & \\
\hline 48 Horas baño & RN con peso $>3500 \mathrm{~g}$ & $\mathrm{Si}$ & 7 & 5 & 201 & 07107 & זמת \\
\hline & & No & 26 & 56 & & & \\
\hline & $\mathrm{RN} \operatorname{con} \mathrm{PC}>35 \mathrm{~cm}$ & $\mathrm{Si}$ & 7 & 3 & & & \\
\hline & & No & 24 & 58 & & & \\
\hline
\end{tabular}

*= p media exacta

Otro aspecto que se valoró en relación con la percepción del dolor perineal post parto, buscaba establecer la eficacia analgésica del Acetaminofén usado en dos dosificaciones diferentes: hasta $2 \mathrm{~g}$ diarios, por una parte, y 3 o $4 \mathrm{~g}$ diarios por la otra.

Si bien no se encontraron diferencias significativas en el nivel promedio de percepción del dolor, si se encontró que dosis de 3 a 4 gramos diarios de acetaminofén se traducen en menores valores promedio de la escala análoga de dolor, tal como se ilustra en la figura 4.

Los valores registrados en la figura 4 sugieren entonces mayor eficacia de dosis altas de acetaminofén en las situaciones que probablemente desencadenen un dolor de tipo agudo (estar sentada o ir al baño). 
Figura 4. Nivel promedio de percepción del dolor postparto según dosis de Acetaminofén

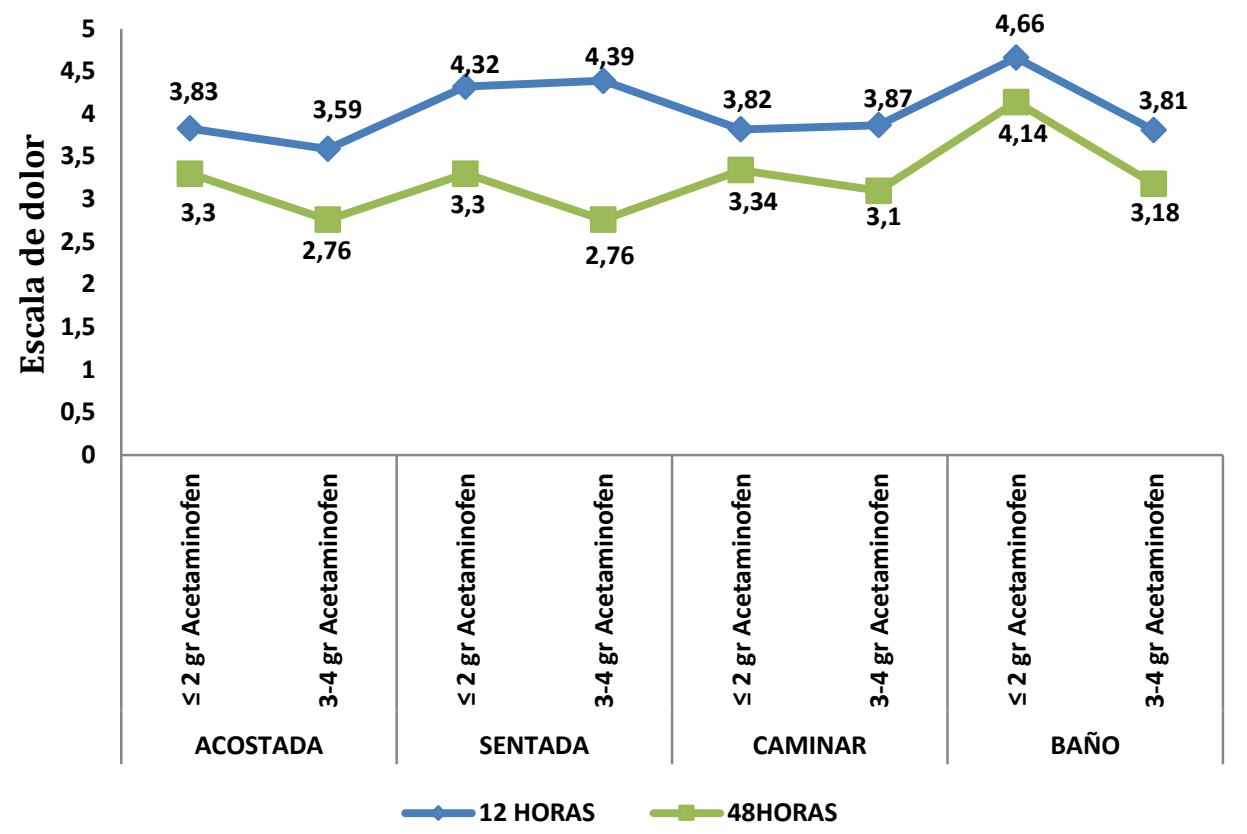

\section{Discusión}

Se registran frecuencias relativamente bajas de dolor perineal postparto en el grupo examinado en comparación con los reportes de otros estudios similares que registran valores de hasta $88,2 \%$ en las primeras 24 horas $^{12}$, superiores incluso al doble de lo reportado en el presente estudio en condiciones de movimiento como el hecho de caminar o de realización de la micción o defecación. Resultado similar se registró en Brasil, en donde la prevalencia de dolor perineal a las 16 horas promedio postparto en primíparas fue de $33 \%$, con una escala media de dolor de $4,5^{13}$.

Acorde con lo reportado en la literatura, la ocurrencia de desgarro grado II o mayor es, después del uso de analgesia peridural durante el trabajo de parto, el factor que mayormente incide sobre la frecuencia e intensidad del dolor perineal en el postparto inmediato. Independiente de las secuelas funcionales que puedan derivarse del manejo que se dé a la lesión, la evidencia estudiada muestra que las mujeres con traumas tipo desgarro grado II o mayor requieren de un manejo analgésico más incisivo y riguroso, así como de seguimiento más detallado ${ }^{14}$.

Los resultados del estudio exploratorio sugieren la necesidad de realizar estudios más detallados sobre la conveniencia del empleo de analgesia epidural durante el período de trabajo de parto con curso normal, habida cuenta de que en este estudio se perfila la circunstancia de que en las mujeres que recibieron la analgesia epidural se registró una mayor intensidad en la percepción del dolor perineal postparto. En otro estudio se encontró que el empleo de Ropivacaína combinada con Fentanyl durante el trabajo de parto se asocia con una mayor duración de la segunda etapa del trabajo de parto y una mayor incidencia de parto asistido y de asfixia neonatal ${ }^{15}$.

Si bien las deficiencias en el manejo adecuado del dolor post-parto pueden constituir un escenario propicio para la ocurrencia de resultados adversos maternos y neonatales, el empleo de analgésicos opioides después del parto vaginal de rutina, para el control del dolor post-parto puede no ser lo más recomendable. Los analgésicos no 
opioides como antiinflamatorios no esteroides (AINE) y acetaminofén, tienen la ventaja de producir menos efectos secundarios ${ }^{16}$. En otras latitudes se está experimentando con técnicas terapéuticas alternativas como el dry cupping, con resultados favorables ${ }^{17}$.

En el ámbito quirúrgico, una dosis única de 2 gr de Acetaminofén por vía intravenosa ha sido empleada rutinariamente en algunos servicios como analgésico en el postoperatorio inmediato de mujeres después del parto por cesárea. Se tiene conocimiento de que la dosis máxima diaria recomendada es de $4 \mathrm{~g}$; el empleo de una dosis de carga de $2 \mathrm{~g}$ IV, seguido de 1 gr cada 6 horas hasta las $24 \mathrm{~h}$, se ha confirmado como seguro y bien tolerado en voluntarios sanos, a la vez que se ha mostrado como un manejo seguro y eficaz en el inmediato alivio del dolor post-parto por cesárea ${ }^{18}$, lo que invita a considerar esta alternativa terapéutica en un futuro estudio clínico.

También es importante considerar que en el análisis de la farmacocinética del Acetaminofén, se ha reportado que en mujeres gestantes ocurre un aclaramiento significativo y mayor del Acetaminofén total, durante el embarazo y en el postparto en comparación con las mujeres no embarazadas ${ }^{19}$, por esta razón, y considerando la posibilidad de riesgo potencial que pueden representar los metabolitos fruto de oxidación del Acetaminofén, se considera importante estudiar las características de estos metabolitos, así como las correspondientes rutas de eliminación durante el parto, por lo cual se recomienda explorar juiciosamente la necesidad del incremento de la dosis en este tipo de pacientes ${ }^{20}$.

\section{Agradecimientos}

Los autores hacen expresa manifestación de gratitud a las mujeres que generosamente ofrecieron voluntad y tiempo para participar en el estudio; también a las directivas y trabajadores del Hospital La Victoria-Instituto Materno Infantil de Bogotá por la colaboración y apoyo durante el desarrollo de este trabajo.

Conflicto de intereses: Ninguno declarado.

\section{Referencias}

1. Marques NA, De Almeida N, Bachion MA, de Souza JT. Concentração plasmática do hormônio adrenocorticotrófico de parturientes submetidas a método não farmacológico de alívio da ansiedade e dor do parto. Rev Latino-am Enfermagem. 2005;13(2):223228.

2. Stuber KJ, Wynd S, Weis KA. Adverse events from spinal manipulation in the pregnant and postpartum periods: a critical review of the literature. Chiropr Man Therap. 2012;20(1):8 doi:10.1186/2045-709X-20-8. Disponible en: http://www.ncbi.nlm.nih.gov/pubmed/22455720 (Cons: 13-03-2014).

3. Macarthur AJ, Macarthur C. Incidence, severity, and determinants of perineal pain after vaginal delivery: A prospective cohort study, Am J Obstet Gynecol. 2004; 191(4):1199-1204.

4. East CE, Sherburn M, Nagle C, Said J, Forster D. Perineal pain following childbirth: Prevalence, effects on postnatal recovery and analgesia usage. Midwifery. 2012;28(1):93.97.

5. Amorim A, Junqueira VO, Barbosa FM, Bick D, González ML. Women's experiences of perineal pain during the immediate postnatal period: A cross-sectional study in Brazil. Midwifery. 2011;27(6):e254-e259. Disponible en: http://www.ncbi.nlm.nih.gov/pubmed/21145149 (Cons: 13-03-2014)

6. Amorim A, Junqueira SM, de Oliveira J, Barbosa FM. Avaliação e tratamento da dor perineal no pós-parto vaginal. Acta Paul Enferm. 2011;24(1):94-100

7. Brasil. Ministério da Saúde. Secretaria de Ciência, Tecnologia e Insumos Estratégicos. Departamento de Ciência e Tecnologia. PNDS 2006: Pesquisa Nacional de Demografia e Saúde da Criança e da Mulher. Relatório. [Internet]. Brasília: Ministério da Saúde; 2008. Disponible

en: http://bvsms.saude.gov.br/bvs/publicacoes/relatorio_ pnds_2006.pdf [Cons: 14-03-2014]

8. Alfonso-Méndez S. Control del dolor y satisfacción de la mujer en el puerperio inmediato. Trabajo de fin de Master universitario en enfermería de urgencias y cuidados críticos. Universidad de Oviedo, España. 2013. Disponible

en: http://dspace.sheol.uniovi.es/dspace/handle/10651/1 7353

9. Smith CA, Collins CT, Cyna AM, Crowther CA. Complementary and alternative therapies for pain management in labour (Review). The Cochrane collaboration 2006. Issue 4. Disponible en: http://onlinelibrary.wiley.com/doi/10.1002/1465185 8.CD003521.pub2/pdf (Consulta: 14-03-2014)

10. Downie WW, Leatham PA, Rhind VM, Wright V, Branco JA, Anderson JA. Studies with pain rating scales. Annals of the rheumatic diseases. 1978;37:378-381

11. Brunelli C, Zecca E, Martini C, Campa T, Fagnoni E, Bagnasco $\mathrm{M}$, et al. Comparison of numerical and verbal rating scales to measure pain exacerbations in patients with chronic cancer pain. Health and quality of life outcomes. 2010;8(1):42. Disponible en: 
http://www.hqlo.com/content/8/1/42 (Cons: 1403.2014)

12. Persico G, Vergani P, Cestaro C, Grandolfo M, Nespoli A. Assessment of postpartum perineal pain after vaginal delivery: prevalence, severity and determinants. A prospective observational study. Minerva Ginecol. 2013; 65(6):669-78.

13. Amorim A, Hiromi M, De Souza C, Luana R, De Paula E, Junqueira SM. Association between perineal trauma and pain in primiparous women. Rev Esc Enferm USP. 2014; 48(Esp):39-44.

14. Leeman LM, Rogers RG, Greulich B, Albers LL. Do unsutured second-degree lacerations affect postpartum functional outcomes?. J Am Board Fam med. 2007;20: 451-457.

15. Li Q, Li CX, Liu Y, Xue WN, Chen TM. Influence of epidural ropivacaine in combination with fentanyl for labor analgesia on the clinical outcome of labor. Nan Fang Yi Ke Da Xue Xue Bao. 2008;28(6):1070-1072.

16. Jones HE, O'grady K, Dahne J, Johnson R, Lemoine L, Milio L, et al. Management of acute postpartum pain in patients maintained on Metadone or Buprenorphine during pregnancy. Am J Drug Alcohol Abuse. 2009;35 (3):151-156.

17. Akbarzade M, Ghaemmaghami M, Yazdanpanahi Z, Zare N, Mohagheghzadeh A, Azizi A. Comparison of the effect of dry cupping therapy and acupressure at BL23 point on intensity of postpartum perineal painbased on the short form of McGill pain questionnaire. J. Reprod. Infertil. 2016;17(1):39-46.

18. Marcus HE, Fabian A, Dagtekin O, Schier R, Krep H, Böttiger BW, et al. Pain, postdural puncture headache, nausea, and pruritus after cesarean delivery: a survey of prophylaxis and treatment.Minerva Anestesiol 2011;77 (11):1043-1049.

19. Kulo A, van de Velde $M$, de Hoon J, Verbesselt $R$, Devlieger R, Deprest J, et al. Pharmacokinetics of a loading dose of intravenous paracetamol post caesarean delivery. Int J Obstet Anesth. 2012;21(2):125-128.

20. Kulo A, Peeters MY, Allegaert K, Smits A, de Hoon J, Verbesselt R, et al. Pharmacokinetics of Paracetamol and its metabolites in women at delivery and postpartum. Br J Clin Pharmacol. 2012;75(3):850-860. 